MYU Tokyo

S \& M 1258

\title{
Design of an Intelligent Vehicle Control Based on the Center of Gravity of Pilot
}

\author{
Chung-Neng Huang* and Chun-Lung Wu \\ National University of Tainan, 33 Sec. 2, Shu-Lin St., Tainan 70005, Taiwan
}

(Received December 21, 2015; accepted May 16, 2016)

Keywords: vehicle control, center of gravity, tricycle, fuzzy control, system stability

Steering wheels and joysticks are presently the most familiar tools for vehicular operations. However, it may become difficult for them to meet special demands, such as those associated with driving in a narrow-and-small capsule, or rapid-responding steering used in entertainment, or by the military. In this study, based on the signals of center of gravity (COG) from the driver's seat, a novel vehicular control method is proposed and developed. In this research, a tricycle system with two front wheels and one rear wheel $(2 \mathrm{~F} / 1 \mathrm{R})$ was designed and set up to simulate the conventional steering mechanism in ships or airplanes. The system primarily consists of a wheel motor, a dc motor and a pilot's seat. They are designed to be a main mover, a steering, and a controller, respectively. In addition, the angles of the slope of the pilot's seat as detected by tilts are used as the displacement signals. The results show that the mean absolute percentage error between the simulation and the experimental studies is smaller than $1.05 \%$. The effectiveness of this proposed design is confirmed.

\section{Introduction}

Vehicle dynamic control by adopting brake-based methods as well as electronic stability programs is generally called a vehicle safety enhanced system. A brake-based vehicle safety enhanced system incorporating the predictability of vehicle behavior based on active control of the individual wheel brake allows a driver to rapidly restore control of a vehicle. However, brake-based safety enhanced systems have drawbacks, such as using the brake force control on each wheel to generate the offset yaw moment or raising the estimated friction coefficient between the tire and the road to control wheel slip. These cause the driving speed to be different from that desired by the driver. ${ }^{(1)}$ On the other hand, there are some advantages in terms of recapturing braking energy and faster response that can be obtained when the vehicle stability enhancement algorithm is controlled by the motor. ${ }^{(2)}$

For people, balancing one's center of gravity (COG) is an innate ability. It is an easier task than learning manual operations, and indeed, no time is required to learn it. Based on this innate ability, we have developed a novel vehicle control method. First, the steering motion control uses gravityshifting signals generated from the driver's seat, and the steering commands are correlated to the tilting of the seat. A wheel motor has been widely used in other studies as the main propulsion for electric vehicles. ${ }^{(3-5)}$ However, the mechanical coupling of a wheel motor is complicated, and it is

"Corresponding author: e-mail: kosono@mail.nutn.edu.tw 
not only difficult to clarify the precise dynamics but also to install a rotational speed sensor. ${ }^{(5)}$ In this study, a model-free mapping technology based on a neural-network (NN) was used for control instead of the mathematical dynamics of a wheel motor. Finally, a fuzzy controller with stable and reliable output performance to deal with real-time speed control ${ }^{(6,7)}$ was adopted to be the input signal processor for this system.

In addition, a control logic for the electrohydraulic brake $(\mathrm{EHB})^{(8)}$ and a rear motor for vehicle stability were proposed to be applied to the EV system of a tricycle. Here, to compensate for the error between the yaw rate and the sideslip angle, a fuzzy control algorithm was adopted to generate the direct yaw moment. The effectiveness of the vehicle stability control was confirmed through MATLAB/Simulink simulations.

\section{Tricycle System Design}

Rear-rudder steering is common in most conventional vehicles such as ships, airplanes, and submarines. To simulate the steering mode of such vehicles, a tricycle system was designed and realized. This system has two front wheels and one rear wheel (Fig. 1), and two tilts are installed below the pilot's seat. In addition, a wheel motor is used as the main driver of the rear wheel. Four springs are installed underneath the seat to increase the damping of the input signals. If the pilot's COG shifts, then the tilts can measure the angle of seat to realize motion control. Because the output signals of the tilts are analog, an $\mathrm{A} / \mathrm{D}$ converter is used to convert the signals into digital ones and transfer them to a microprocessor to control the movement. In addition, a dc motor as a steering driver is mounted on the rear wheel. This uses a gear to change the direction of the vehicle.

\subsection{Evaluation of system stability}

In Fig. 2, a two-degree-of-freedom vehicle model is employed as the control platform for the design of the yaw moment controller. The terms $x, y$, and $z$ are the longitudinal, lateral, and vertical directions, respectively; $m$ and $v$ are the vehicle mass and velocity; $F$ is the tire force; $\beta$ is the sideslip angle; $\gamma$ is the yaw rate; $L$ is the wheel base; $I_{z}$ is the inertia moment; $M$ is the tire force at each wheel generating the direct yaw moment; $W$ is the vehicle tread; $f r$ and $f l$ are the right and the left wheels in front; and $r$ is the rear wheel. The equations governing the motion directions in longitude, lateral, and yaw are described as follows:

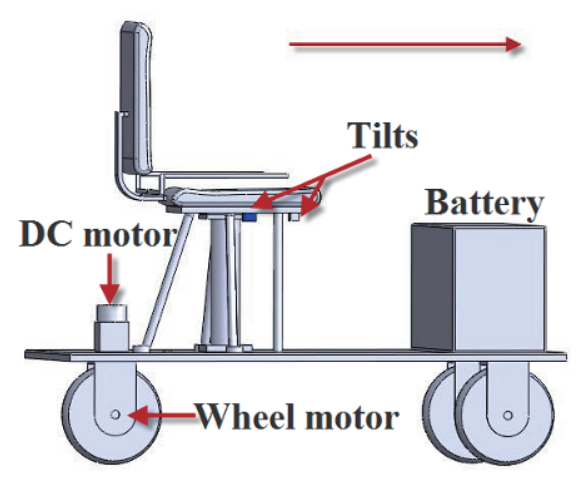

Fig. 1. (Color online) Tricycle structure. 


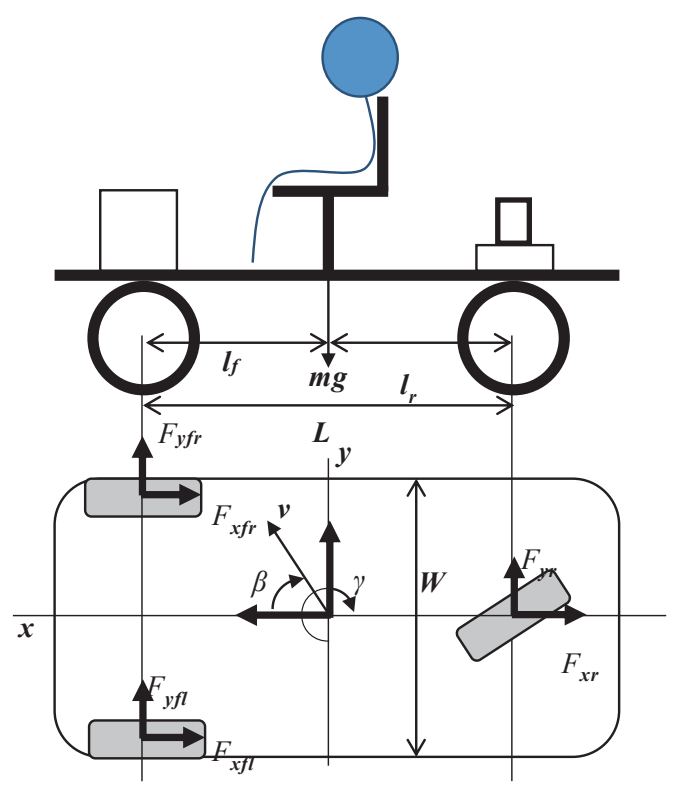

Fig. 2. (Color online) Vehicle motion and parameters.

Longitudinal motion:

$$
m \dot{V}=\Sigma F_{x}=F_{x f r}+F_{x f l}+F_{x r}
$$

Lateral motion:

$$
m V(\dot{\beta}+\gamma)=\Sigma F_{y}=F_{y f r}+F_{y f l}+F_{y r}
$$

Yaw motion:

$$
\begin{gathered}
I_{z} \dot{\gamma}=\Sigma M_{z}=\left(F_{x f r}+F_{x f f}\right) \times L_{f}-F_{x r} \times L_{r}+M \\
M=-\frac{W}{2}\left(F_{x f r}-F_{x f f}+F_{x r}\right)
\end{gathered}
$$

In braking, the wheel slip ratio $\lambda$ is calculated as

$$
\lambda=\frac{R \omega-v}{v}
$$

where $R$ is the effective rolling radius of the wheel, and $\omega$ is the angular velocity of the wheel.

The longitudinal tire forces can be calculated using

$$
F_{x^{*}}=k_{u} \lambda F_{z}
$$

Here, $k_{u}$ is the slope of the tire characteristics from the linear region of a $\mu-\lambda$ curve, and the lateral tire forces are linearized with the slip angles: ${ }^{(9)}$

$$
F_{y f r}+F_{y f t} \approx-C_{f} \alpha_{f},
$$




$$
F_{y r} \approx-C_{r} \alpha_{r}
$$

For the axle, the positive cornering stiffness $C_{f}$ and $C_{r}$ of front and rear tires are twice that of a single tire. By using Eqs. (1)-(8), the state equations of the system can be expressed as follows:

$$
\begin{gathered}
\dot{x}=A x+B u+E r, \\
A=\left[\begin{array}{cc}
-\frac{C_{f}+C_{r}}{m v} & -1-\frac{l_{f} C_{f}-l_{r} C_{r}}{m v^{2}} \\
-\frac{l_{f} C_{f}-l_{r} C_{r}}{I_{z}} & \frac{l_{f}^{2} C_{f}+l_{r}^{2} C_{r}}{I_{z} v}
\end{array}\right], \\
B=\left[\begin{array}{c}
0 \\
\frac{W k_{u} F_{z}}{2 I_{z}}
\end{array}\right], \\
E=\left[\begin{array}{cc}
\frac{C_{f}}{m v} & 0 \\
\frac{l_{f} C_{f}}{I_{z}} & \frac{1}{I_{z}}
\end{array}\right],
\end{gathered}
$$

where $F_{z}$ is the function of a static vertical load, and $\alpha_{r}$ is the steering angle of rear wheel.

By substituting the specification data of the case study vehicles in Ref. 10 and the physical data measured from the tricycle platform, the root locus of this system with respect to a controller gain of $K=0$ to 400 was plotted and is shown in Fig. 3. This plot demonstrates that, as the controller gain $K$ increases, the roots move to the left-half plane.

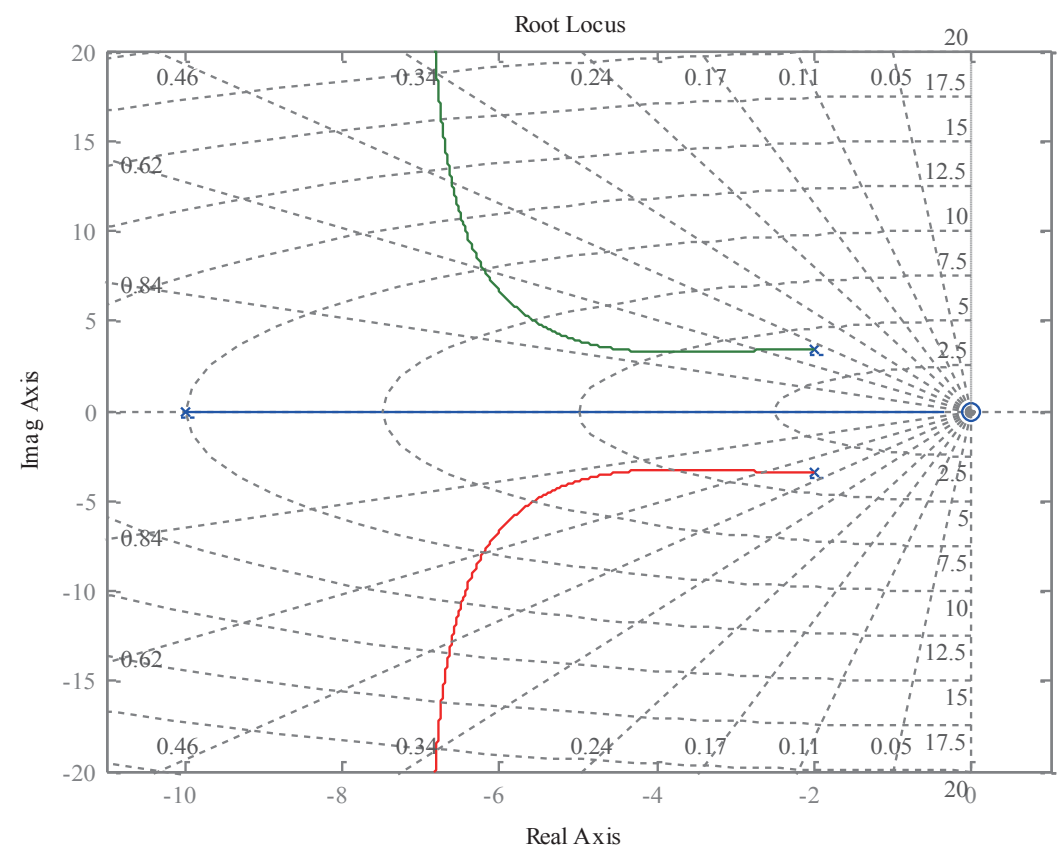

Fig. 3. (Color online) Proof of system stability. 


\subsection{Control algorithm design}

As the desired path, the driver turned a steering angle $\delta$; then the tilts detected the actual sideslip angle $\beta$ and yaw rate $\gamma$ which were compared with the desired sideslip angle $\beta_{\mathrm{d}}$ and yaw rate $\gamma_{\mathrm{d}}$ found using Eq. (9). Here, $\beta_{\text {error }}$ and $\gamma_{\text {error }}$ were employed as the input variables of the fuzzy controller.

The fuzzy controller was designed as a 2-input-and-2-output system. A fuzzy controller is based on the human brain which handles inexact information which is difficult to express precisely by mathematical models. Fuzzy systems that combine fuzzy sets with fuzzy rules to form numerical estimators can describe overall complex nonlinear behavior. This concept has been successfully applied to many commercial products and industrial systems, most notably to automated control, management science, and artificial intelligence. Reference 11 discussed a tracking problem for a tricycle mobile robot with two degrees of freedom and subject to the front wheel steering which was solved using a fuzzy logic control. In Ref. 7, a fuzzy control system was designed for an autonomous robot cart operating in an unknown environment which was unpredictable and dynamic. In this study, based on an intelligent digital redesign method, the stability analysis for the fuzzy system was proposed as

$$
\begin{gathered}
\operatorname{shift}(k)=y_{r}(k)-y(k), \\
\Delta \operatorname{shift}(k)=\operatorname{shift}(k)-\operatorname{shift}(k-1),
\end{gathered}
$$

where $\operatorname{shift}(k)$ and $\Delta \operatorname{shift}(k)$ are the input angle of body slope and the last of location, respectively; $y_{r}$ is the input reference; and $y$ is the system output.

In Fig. 4, the membership function was employed to convert the input and output variables into linguistic ones for fuzzy control. The scaling factors $S_{i}$ with the subscript $i=1,2,3$ represent the output error, the change error of the system, and the control output. ${ }^{(11)}$ Table 1 shows the control rules for fuzzy decision.

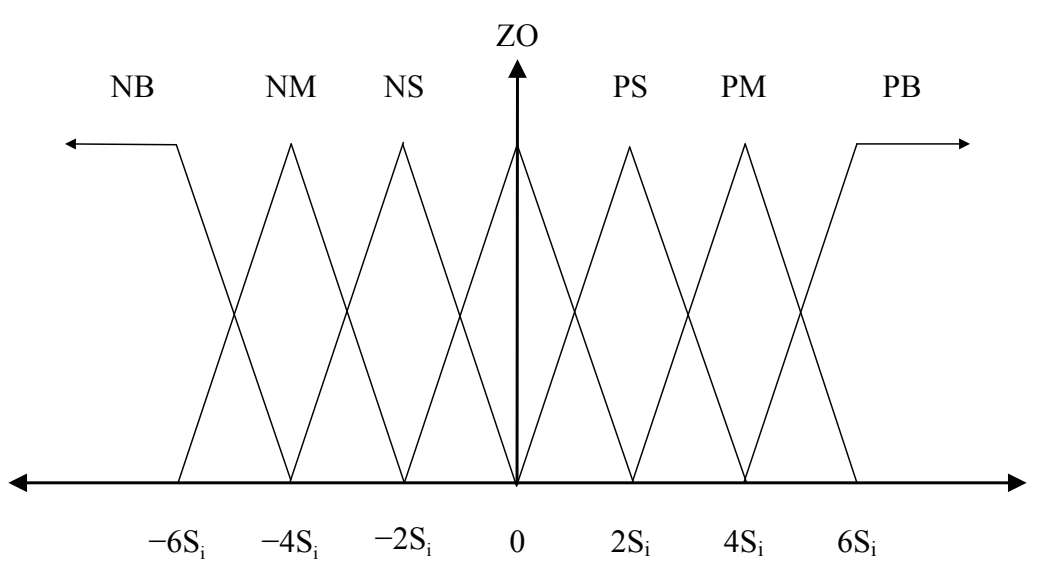

Fig. 4. Membership function of fuzzy controller. 


\section{Simulation Studies and Confirmation}

To confirm the effectiveness of the proposed control, a case study was designed and a physical test on the experimental platform was conducted.

\subsection{Case study}

In Fig. 5, a case study is shown. The vehicle model was designed and plotted in Cartesian coordinates which represent the vehicle position. The following symbols are used: $O$ is the present position and the center of rotation of the vehicle, $\overline{Q P}$ is the radius of desired path, $P^{*}=\left(x_{p}, y_{p}\right)$ is the desired position, $V$ is the initial speed of vehicle $(5 \mathrm{~km} / \mathrm{h})$, and $\alpha$ is the steering angle, and $\overline{O x_{p}}$ is the minimum difference in distance between the current position and a desired path.

\subsection{Results of the simulation and discussion}

Based on the setup described, the results with respect to driver mode and proposed control were obtained and are shown in Fig. 6, where the vertical axis indicates the approaching movement of the vehicle along the $x$-axis toward desired point $P^{*}$. The horizontal axis is time in seconds. When the

Table 1

Fuzzy rule table. ${ }^{(11)}$

\begin{tabular}{lccccccc}
\hline \multirow{2}{*}{$\Delta$ shift $(k)$} & \multicolumn{7}{c}{ shift $(k)$} \\
\cline { 2 - 7 } & NB & NM & NS & ZO & PS & PM & PB \\
\hline NB & PM & PS & PS & ZO & PS & PM & PB \\
NM & PS & PS & PS & ZO & PS & PM & PM \\
NS & PS & PS & PS & ZO & PS & PS & PS \\
ZO & ZO & ZO & ZO & ZO & ZO & ZO & ZO \\
PS & NS & NS & NS & ZO & NS & NS & NS \\
PM & NM & NM & NS & ZO & NS & NS & NS \\
PB & NB & NM & NS & ZO & NS & NS & NM \\
\hline
\end{tabular}

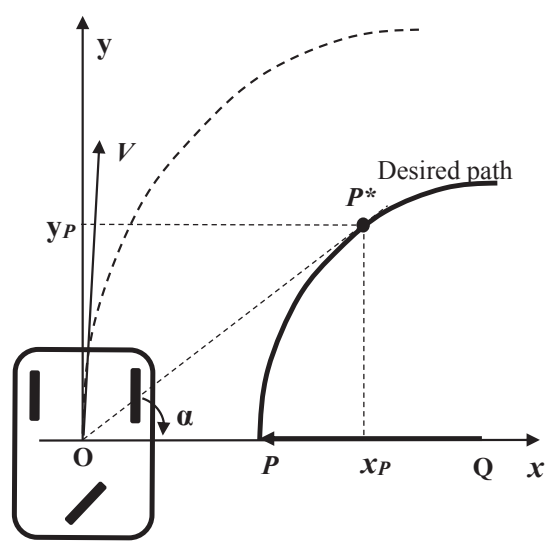

Fig. 5. Case study.

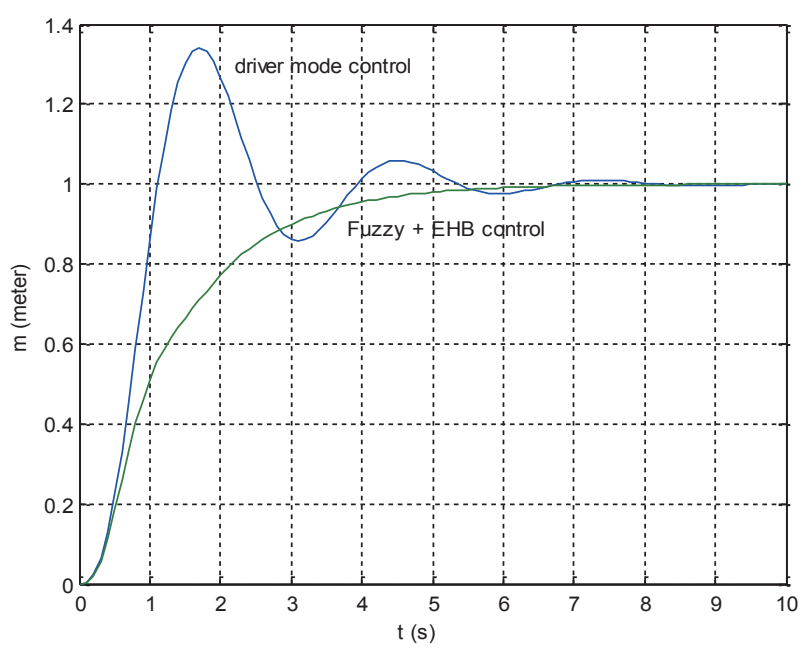

Fig. 6. (Color online) Control results. 
steering operation was under driver control, a maximum deviation of $0.35 \mathrm{~m}$ occurred in $1.75 \mathrm{~s}$, and approximately $9 \mathrm{~s}$ was required to approach the desired path. On the other hand, when the vehicle was under the proposed control and approached within a distance of $0.2 \mathrm{~m}$ from the desired position on the $x$-axis, the speed was slowed due to the control by the EHB. This resulted in no deviation occurring under the control. In addition, the vehicle approached the desired position in $6.5 \mathrm{~s}$, which was faster by $2.5 \mathrm{~s}$ than the one under driver mode.

\section{Conclusions}

In this study, a vehicle control method based on the COG of the pilot was proposed and developed. Using a tricycle $(2 \mathrm{~F} / 1 \mathrm{R})$ model, the system dynamics were derived and system stability was demonstrated. Fuzzy and EHB controls were integrated to improve the system stability. The control effectiveness and feasibility of the proposed method and design were investigated and confirmed through simulations on the system platform. The success of this study shows that the proposed system and control method can not only replace existing controls but also improve the control of manned vehicles.

\section{Acknowledgements}

This work was supported by the National University of Tainan under Grant AB104-124.

\section{References}

1 K. Kin, O. Yano, and H. Urabe: JSAE Rev. 24 (2003) 71.

2 S. Sakai, H. Sado, and Y. Hori: IEEE/ASME Trans. Mechatron. 4 (1996) 9.

3 Y.-P. Yang and D. S. Chung: IEEE Trans Magn. 43 (2007) 51.

4 Y.-P. Yang, J.-P. Wang, S.-W. Wi, and Y.-P. Luh: IEEE Trans. Magn. 40 (2004) 1883.

5 K. Cakir and A. Sabanovic: Proc. IEEE Int. Conf. Advanced Motion Control (IEEE, Istanbul, 2006) pp. 613618.

6 R.-E. Precup and S. Preitl: Proc. IEEE Int. Conf. Mechatronics, (IEEE, Budapest, 2006) pp. 203-208.

7 O. Farhi and Y. Chervenkov: Proc. IEEE Int. Conf. Intelligent Systems (IEEE, Varna, 2008) pp. 4-62-4-65.

8 K. Park and S.-J. Heo: Int. J. Veh. Des. 36 (2004) 38.

9 Y. Hori, Y. Toyoda, and Y. Tsuruoka: IEEE Trans. Ind. Appl. 34 (1998) 1131.

10 E. Esmailzadeh, A. Goodarzi, and G. R. Vossoughi: Mechatronics 13 (2003) 659.

11 R.-E. Precup and S. Preitl: Acta Polytech. Hung. 3 (2006) 61. 